\title{
SOLAR SIMULATOR ON THE BASIS OF POWER- FUL LIGHT-EMITTING DIODES
}

\author{
Oxana Aldoshina ${ }^{1 *}$, Vyacheslav Yugay ${ }^{2}$, Nurbol Kaliaskarov², Ulan Esenjolov², Saltanat \\ Nesipova $^{2}$ \\ ${ }^{1}$ National Research Tomsk Polytechnic University, Lenin Avenue, 2, 634050, Tomsk, Russia \\ ${ }^{2}$ Karaganda State Technical University, 56, Mira, Karaganda, 100000, Kazakhstan
}

\begin{abstract}
The Article describes the construction design and experimental results of the Solar Simulator, based on powerful LEDs with different radiation spectrum. There was established that the block from 25 light-emitting diodes provides radiation intensity sufficient for carrying-out of AM1.5G standard requirements. Fine tuning of the resultant spectrum and the required illumination for all radiation spectral components were obtained through light-emitting diodes current control.
\end{abstract}

\section{The object of study}

Solar Simulators provide illumination that is similar to natural sunlight in accordance with the requirements of the standard. They are intended for solar battery parameters estimation. Conditions for solar spectrum AM1.5 are more often used during the simulation operation.

There are several types of sources of light that are used for implementation of Solar Simulators. Xenon arc lamps are the most commonly used source of flat spectrum for today. There is a need of complicated optical system for this source that should provide a steady light during the testing and leads to low Energy Performance of this test system. Recently the medium power light-emitting diodes (around 1.5W) have been already applied for sunlight simulation, which intensity has $90 \%$ of the required standard intensity [1].

In this Article, we study and evaluate the features of Solar Simulators based on modern high power LEDs. In addition, there was conducted research of hybrid LEDs and sources in the form of halogen lamps with a B class spectrum usage [2].

Solar Simulator was designed with the use of light-emitting diodes, which are set to the printed wire board in the form of hexagon-shaped star. Most of the radiant power, about 10W, is formed by Bridgelux white light-emitting diodes. These light-emitting diodes provide necessary emission in $500 \ldots 600 \mathrm{Nm}$ spectrum and produce main part of the required optical power for $400 \ldots 500 \mathrm{Nm}$ spectral band $[3,4]$.

The rest of power $3 \mathrm{~W}$ is produced by Luxeon blue light-emitting diodes. LedenginLZ4 $10 \mathrm{~W}$ light-emitting diodes were used as a light source in 600-700 Nm and 700-800 Nm wavelength band, while Osram IR LEDs were used in 800-900 Nm and 900-1100 Nm band. Picture 1 shows the radiation spectrum of the used LEDs.

\footnotetext{
* Corresponding author: oxi80-07@mail.ru
} 


\section{Development}

Printed wire boards with light-emitting diodes were placed in "comb" form, in order to provide optimal flow homogeneity, and set on the aluminum square plate, which was installed on the blown aluminum radiator (Figure 1). Each color of the radiation spectrum was regulated by an independent current, which was provided by the switched power supply TTI QL564-TP and Mastech HY-5003.

The spectral distributions of the power of the LEDs were measured by multichannel analyzer Hamamatsu PMA-12 and a spectrometer AvantesAvaSpec 2048 (Figure 2).

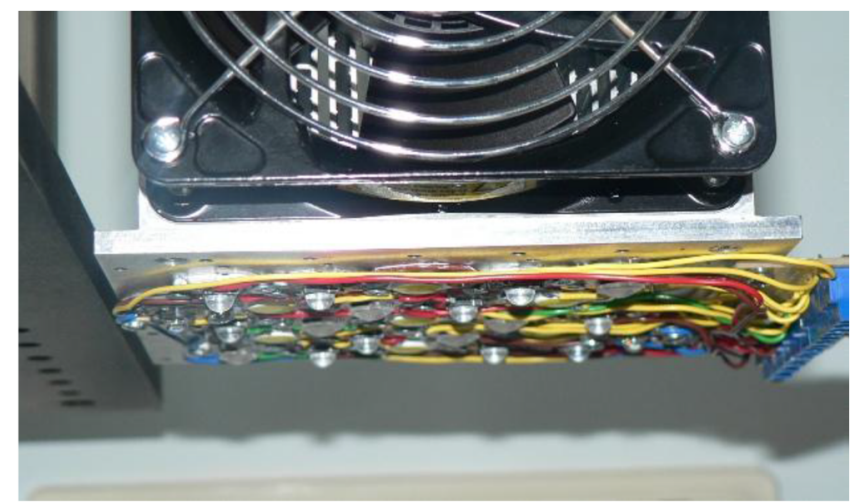

Fig. 1. Matrix of LEDs mounted on an aluminum plate with a radiator.

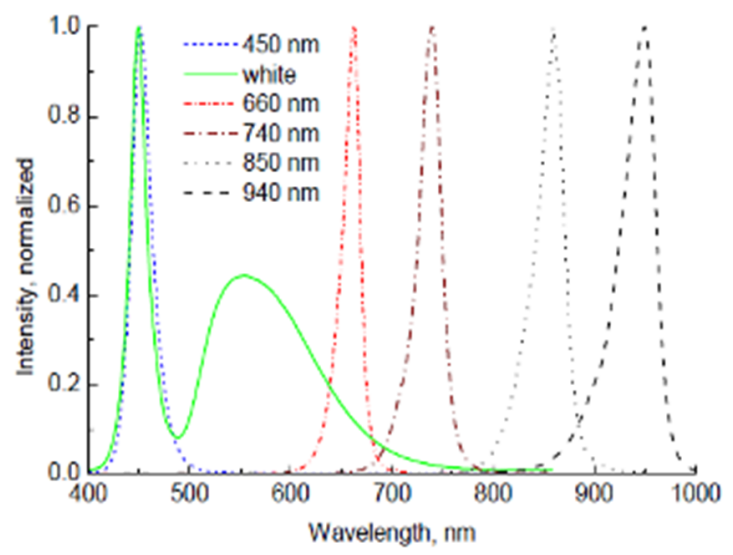

Fig.2. Radiation spectra of used LEDs.

The body of the Simulator was focused on the carrying-out of the AM1.5 requirements. Spectral and power characteristics of the Simulator with the selected light-emitting diodes were represented in Table 1. Six spectral bands and required illumination band were shown in the first two table columns. Prospective powers of the light-emitting diodes in the six spectral bands were shown in the right columns. The required illumination of each diodes group was determined on this value basis (the third line from below). After multiplying of this result to the projected sensitivity of the testing silicon photodiode (second line from below), the required photocurrent density came out, which was shown in the last line of the Table 1. 
Table 1. Spectral and power characteristics of the Simulator.

\begin{tabular}{|c|c|c|c|c|c|c|c|}
\hline Range, $\mathrm{nm}$ & \multirow[t]{2}{*}{$\mathrm{mW} / \mathrm{cm}^{2}$} & \multicolumn{6}{|c|}{ The power of LEDs in the spectral ranges (\%) } \\
\hline & & $450 \mathrm{~nm}$ & white & $660 \mathrm{~nm}$ & $740 \mathrm{~nm}$ & $850 \mathrm{~nm}$ & $940 \mathrm{~nm}$ \\
\hline $400-500$ & 18.4 & 93 & 33 & - & - & - & - \\
\hline $500-600$ & 19.9 & 3 & 44 & - & - & - & - \\
\hline $600-700$ & 18.4 & - & 23 & 99 & 6 & - & - \\
\hline $700-800$ & 14.9 & - & & - & 98 & 7 & - \\
\hline $800-900$ & 12.5 & - & & - & - & 6 & 5 \\
\hline $900-1100$ & 15.9 & - & & - & - & - & 97 \\
\hline $\begin{array}{l}\text { Required } \\
\mathrm{mW} / \mathrm{cm}^{2}\end{array}$ & illumination, & 3.7 & 46.0 & 9.1 & 152 & 13.4 & 15.4 \\
\hline $\begin{array}{l}\text { Estimated* } \\
\mathrm{W}\end{array}$ & nsitivity, A / & 0.5 & 0.5 & 0.6 & 0.7 & 0.68 & 0.57 \\
\hline $\begin{array}{l}\text { Photocurrer } \\
/ \mathrm{cm}^{2}\end{array}$ & ensity, $\mathrm{mA}$ & 0.8 & 19.0 & 4.9 & 9.1 & 8.3 & 9.0 \\
\hline
\end{tabular}

* Approximate values based on tabular data.

\section{Measurement results}

It was further investigated the distribution of radiation flux at various distances using motorized 2D platform (stands 8MT195 and 8MT295) and photodiode (OsramBPW34B) a Thorlabs power meter(PM100D). In Figure 3 and Figure 4 presents the results of experimental determination of the intensity of light radiation at a distance of $12 \mathrm{~cm}$ from the plane of installation of the simulator.
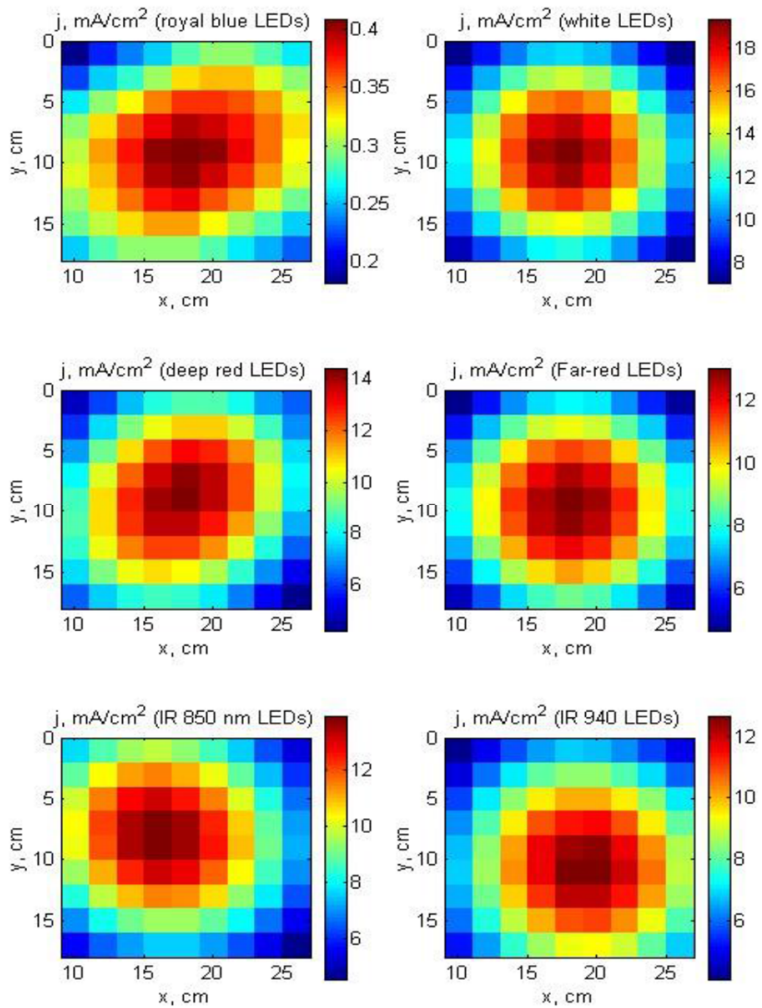

Fig. 3. Dependence of the photocurrent induced by a silicon photodiode OsraBPW34B at the measurement point. 
The photocurrent of the photodiode Osram BPW34B Si was measured in two positions at a distance of $2 \mathrm{~cm}$ from each other. Arrays of various colors are switched one after the other in order to assess whether sufficient photocurrent for the illumination.At a distance of $12 \mathrm{~cm}$ from the plane of installation of the led array white led generates a current density of more than $18 \mathrm{~mA} / \mathrm{cm} 2$.In accordance with the requirements AM1.5G it is a necessary level of illumination in the appropriate spectral range. The excess radiation power present in all of the above light-emitting diodes with a wavelength of about $600 \mathrm{~nm}$. The contribution of blue LEDs is not sufficient for perfect compliance AM1.5G.However, they are not allowed by the standard, required for the spectrum in the range of 0.75 to 1.25 .

As can be seen from Figure 4, from all of the LEDs operating at maximum rated current, photocurrent of the silicon photodiode exceeds $70 \mathrm{~mA} / \mathrm{cm} 2$.From this result follows that in this case, the photon flux is much higher than required in the condition of AM1.5G.

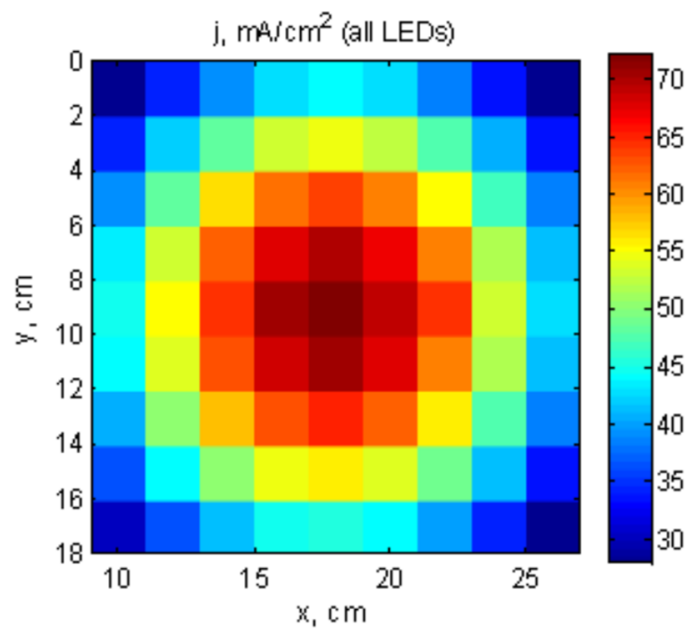

Fig. 4. Dependence of the photocurrent induced by the silicon photodiode OsramBPW34B in the case when all the LEDs operate at a rated current.

The peak of illumination of the developed solar's simulator was measured for all six LED groups. The summarized results of the measurements are presented in Figure 5.

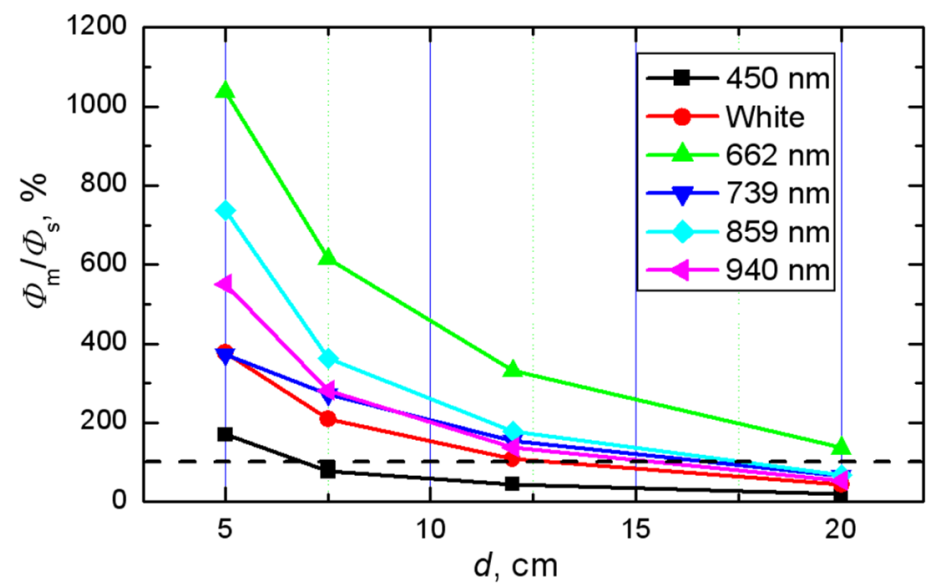

Fig. 5. Peak of illumination depends on distance to simulator. 
For the most powerful diode group (white color) the required peak light exceeded respectively 4 and 2 times. Large excess capacity also has for the diode groups with wavelengths above $600 \mathrm{~nm}, 450 \mathrm{~nm}$. These LEDs exhibit lower peak illumination, however, the main contribution in the wavelength range $400-500 \mathrm{~nm}$ is provided by white LEDs.

During the study of the illumination parameters and the spectral homogeneity of the simulator, the optimal distance was determined experimentally, which was approximately 8.2 $\mathrm{cm}$ from the lens of the LEDs. For the best approximation to the requirements of AM1.5G, optimal LED currents were also found. The spectral inhomogeneity of illumination at a higher spatial resolution was determined using a crystalline silicon photodiode. The result is shown in Figure 6.

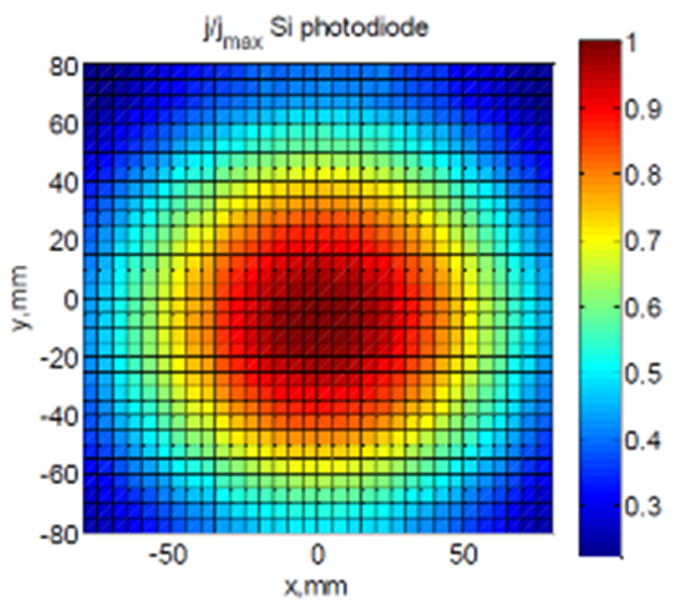

Fig. 6. Photocurrent distribution at a distance of $8.2 \mathrm{~cm}$ for a crystalline silicon photodiode detector.

For a circular area with a diameter $2 \mathrm{~cm}$ deviation of the photocurrent is less than $2 \%$, which corresponds to the A-class heterogeneity. For B and C classes, this area grows up to 3 $\mathrm{cm}$ and $4 \mathrm{~cm}$, respectively.

\section{Conclusion}

To build a solar simulator of A class that meets the requirements AM1.5G, it is sufficient to use the 6 kinds of high power LEDs ( $10 \mathrm{~W}$ or more than 5 groups, 3 watts each).

In the developed simulator to produce the desired illumination of the usable area in the form of a circle with a diameter of several centimeters is used 25 LEDs and 39 watts of electric power.

\section{References}

[1] S.H. Jang, M.W. Shin, Current Applied Physics 10, 537 (2010).

[2] M. Bliss et al., Solar Energy Materials \& Solar Cells 93, 825 (2009).

[3] A. Okhorzina, A. Bikbulatov, A.Yurchenko, N. Bernhard, O. Aldoshina, MATEC Web of Conf. 79, 01010 (2016)

[4] E V Yurchenko, A D Mehtiev, V V Yugai, F N Bulatbayev. IOP Conf. Series: Materials Science and Engineering 81 (2015) 012114 doi:10.1088/1757-899X/81/1/012114 